\title{
Adaptive Energy-Efficient On-Demand Distance Vector Routing Protocol for MANET
}

\author{
Badal Master \\ K.J.Somaiya COE \\ Vidyavihar, Mumbai
}

\author{
Prasanna Shete \\ K.J.Somaiya COE \\ Vidyavihar, Mumbai
}

\begin{abstract}
A Mobile Ad-Hoc Network (MANET) is a collection of wireless mobile nodes forming a temporary network without using any centralized access point, infrastructure, or centralized administration.

The proposed Adaptive Energy-efficient On-demand Distance Vector routing (AEODV) Protocol for MANETs that is based on the Ad-hoc On-demand Distance Vector (AODV). The AEODV makes significant reduction in the energy consumption of the mobile nodes batteries for large no of connections. The balanced energy consumption is done by SARSA Reinforcement learning. At route discovery due to link breakage route is maintained using available backup routes instead of flooding control packets to the whole network area. It saves significant amount of energy which is consumed by rebroadcasting.
\end{abstract}

To show the efficiency of the proposed protocol, it simulated using QualNet. Simulation results show that the AEODV protocol makes an improvement in delay, throughput, total packets received and residual energy compared to AODV and SARSA.

\section{General Terms}

Wireless Mobile Ad-hoc Network, Computational Intelligence, Energy-efficient.

\section{Keywords}

MANET, Reinforcement learning, SARSA online Policy, Route Maintenance

\section{INTRODUCTION}

In the wireless ad-hoc networks, there is a necessity to deploy path for independent mobile users. There are various applications for such networks: emergency/rescue operations, disaster relief efforts, and military networks. There is no centralized control to manage it and can be behaved as applications of Mobile Ad Hoc Networks [1]. A MANET is an autonomous collection of mobile users that communicate over relatively bandwidth constrained wireless links. There are rapid changes in topologies due to mobility unpredictably over time. The network is decentralized; where all network activity including discovering the topology and delivering messages must be executed by the nodes themselves.

Energy-efficient communication is critical for increasing the life of power limited wireless ad hoc networks. Each of the mobile nodes is operated by a limited energy battery and usually it is impossible to recharge or replace the batteries during a mission. Since wireless communications consume significant amounts of battery power, therefore the limited battery lifetime imposes a severe constraint on the network performance. The Nodes are battery-powered, thus energy is a precious resource that has to be carefully used by the nodes in order to avoid an early network partition and hence the study and implementation of energy- efficient algorithms for wireless networks quite constitutes a vast area of research in the field of ad hoc networks.

\section{RELATED WORK}

To minimize energy consumption, many energy-efficient routing protocols have been proposed [2], [3], [4], [5], [6], [7], [8], [9],[10]. These protocols are following classified into two categories: Minimum Energy routing protocols [2], [3], [4], [5], [6], [7] and Maximum Network Lifetime routing protocols [8], [9], [10]. Minimum Energy routing protocols discover for the most energy-efficient route from the source to the destination, while Maximum Network Lifetime routing protocols try to balance the residual battery-power at each node when discovering for the energy-efficient path.

\section{PROPOSED PROTOCOL AEODV}

The AEODV consumes balanced energy among all mobile nodes. To enable mobile nodes to learn an adaptive energyaware RREQs forwarding policy. This is achieved by using the Reinforcement Learning [11] framework.

The another path will be taken if established path's link breaks. It will reduce energy consumption by eliminating issues of conventional AODV [12] protocol to rebroadcast frequently due to broken link.

There are two phase to reduce energy consumption:

- Control RREQ by using Energy-Aware SARSA Algorithm [10]

- Multi-path Route Maintenance to eliminate Re-route Discovery [13]

\subsection{Control RREQ using Energy-Aware SARSA Algorithm}

Using Reinforcement Learning each node learn appropriate forwarding rate which presents its desire to participate in routes discovery process. The goal is to balance energy consumption among mobile nodes and thus increasing the overall network lifetime. This is done by the following Reinforcement Learning model:

The set of States: the mobile node state at time step t, St, denotes its residual lifetime (in seconds) evaluated as follows:

$$
\text { St }=\frac{\text { REt }}{\text { DRatet }}
$$

Where REt and DRatet describe, respectively, the residual energy and the energy drain rate at time step t. To measure the energy drain rate per second, each node monitors its energy consumption during a $\mathrm{T}$ seconds sampling interval.

The state space is experimentally quantized into discrete intervals so that: 
St E $\{[0,500),[500,1000),[1000,1500),[1500,2000),[2000$, INF) $\}$ and $|S|=5$. A high state-value indicates that the current node have a high expected lifetime and vice versa.

The set of actions: A node take decision based on the ratio of RREQs to forward by picking an action in the set: $A=\{0,0.2$, $0.4,0.6,0.8,1\}$, where $|\mathrm{A}|=6$.

The Reward Calculation: the reward is to enhance balanced energy consumption among live nodes. The idea is that a positive reinforcement is given to node with an energy drain rate that is lower than the others and vice versa. Furthermore, the reinforcement value is made proportional to node residual energy.

The reward is calculated by following procedure. Each node adds its drain rate to each forwarded RREQ. When RREQ receives at the destination node, each RREQ packet will contain the sum of drain rates of every node along path. The corresponding RREP, replied by the destination node, will carry the average of drain rates, Avrg_DRate, obtained by dividing the received sum on the RREQ' hops number. The intermediate node, calculates deviation of drain rate dvt by following equation:

$$
\mathrm{dvt}=\frac{\sum_{\mathrm{i}=1}^{\mathrm{N}} \mathrm{AVG} \mathrm{BRR}_{\mathrm{atet}}}{\mathrm{N}}-\text { DRatet }
$$

Where $\mathrm{N}$ is the number of RREPs arrived at intermediate node. The reinforcement reward $\mathrm{rt}$ is given by (3). Note that no reward is calculated when $\mathrm{dVt}$ or $\mathrm{N}$ are not available.

$$
\mathbf{R t}=\left\{\begin{array}{c}
\frac{R E t}{d v t}, \quad d v t>0 \\
\frac{C E t}{d v t}, d v t<0 \\
0, d v t=0 \text { or } N=0
\end{array}\right.
$$

In (3), REt and CEt denote, respectively, the residual energy and the consumed energy at time-step t.

The Q-value calculation: Whenever the reward signal is calculated, than the state-action value $\mathrm{Q}(\mathrm{s}, \mathrm{a})$ at an intermediate node is updated according to the SARSA RL algorithm as in (4).

$\mathbf{Q}(\mathbf{S t}$, at $) \leftarrow \mathbf{Q}(\mathbf{S t}$, at $)+\alpha[\mathbf{r t + 1}+\gamma \mathbf{Q}(\mathbf{S t}+\mathbf{1}, \mathbf{a t}+\mathbf{1})-\mathbf{Q}(\mathbf{S t}$, at $)]$ (4)

Balance in exploration and exploitation is achieved by the $\varepsilon$ greedy action selection rule.According to rule, the RL agent selects with a probability, $1-\varepsilon$, the best action, and selects uniformly with a probability, $\varepsilon$, and an action at random.

Initializations of Q-value:

Initialize $\mathrm{Q}$ (st, at) for each states and actions;

Initialize St;

Choose an action at (a forwarding-policy) using $\varepsilon$-greedy policy derived from Q;

Repeat for each time-step:

1. Take action at to forward RREQ packet;

2. Observe the reward $\mathrm{rt}+1$ and the state $\mathrm{St}+1$ when RREP arrives at Intermediate node;

3. Choose an next action at +1 using $\varepsilon$-greedy policy derived from Q:

4. Update $\mathrm{Q}(\mathrm{St}, \mathrm{at})$ :

$\mathrm{Q}(\mathrm{St}, \mathrm{at}) \leftarrow \mathrm{Q}(\mathrm{St}, \mathrm{at})+\alpha[\mathrm{rt}+1+\gamma \mathrm{Q}(\mathrm{St}+1, \mathrm{at}+1)-\mathrm{Q}(\mathrm{St}, \mathrm{at})]$

5. $\mathrm{St} \leftarrow \mathrm{St}+1$;
6. at $\leftarrow$ at +1 ;

Until the terminal sate is reached (the node energy is exhausted).

\subsection{Multipath Route Maintenance to eliminate Re -Route Discovery}

Each node accepts same RREQ packet from multiple neighbors and creates multiple routes from source to itself. These routes work as backup routes for link breakage issue.

According to mechanism after establishing route, if link breaks then downstream node of broken link sends route maintenance packet towards destination. Any node along path to destination has another path for source to destination, then data will be transmitted by another path.

Flowchart for this mechanism is described in Figure 1.

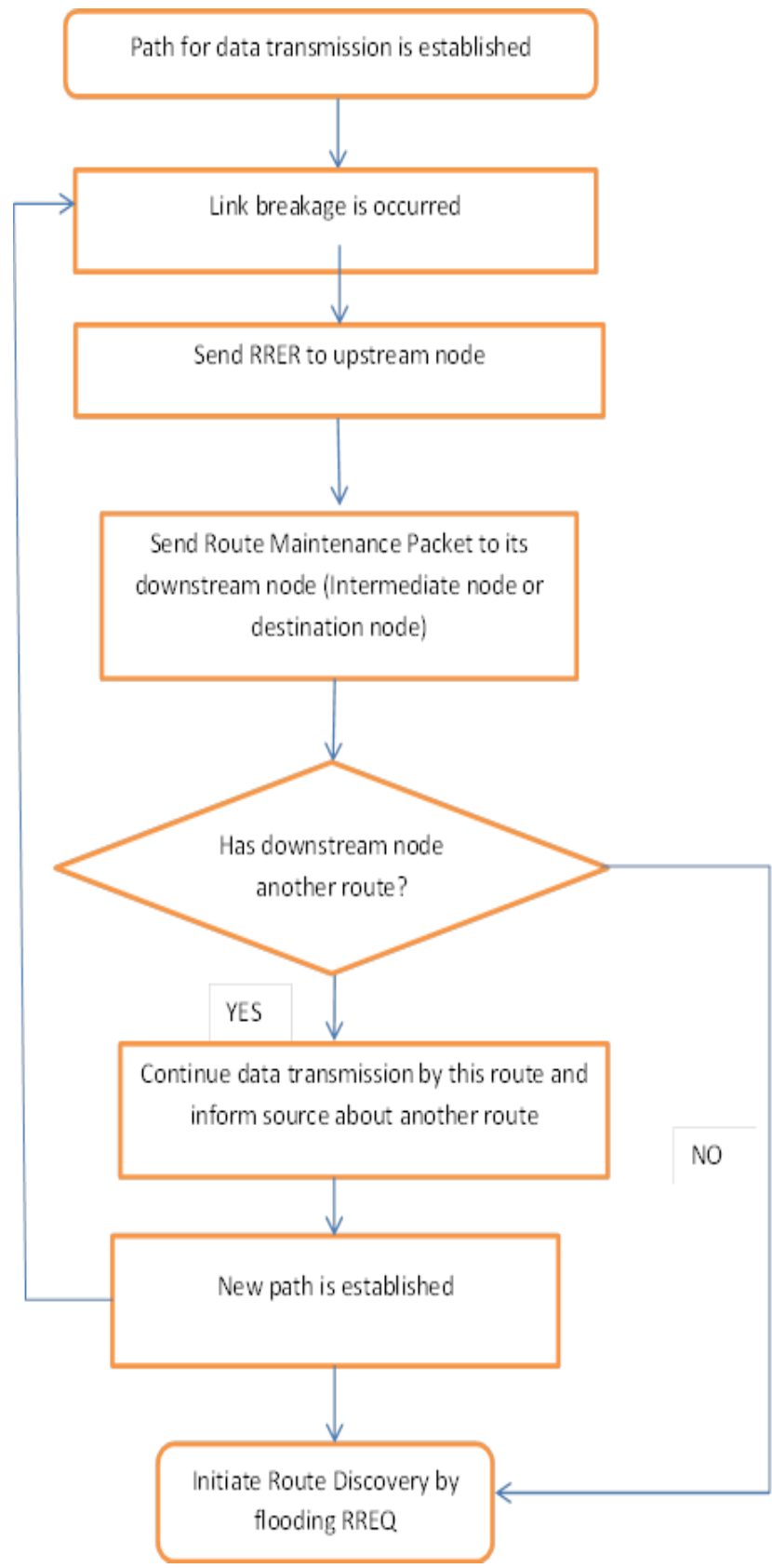

Figure 1

The node receives same RREQ packet (RREQ 1 and RREQ 2). It stores multiple path is described in figure 2. 


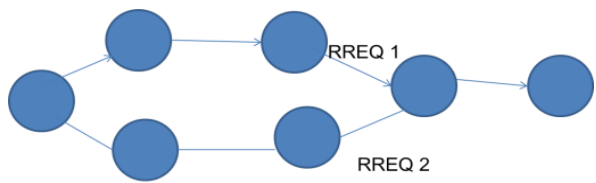

Figure 2

Figure 3 depicts that when route breakage is occurred along established path which is described at red lined active path.

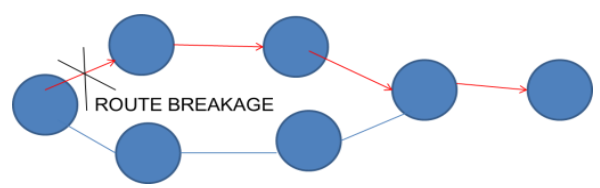

Figure 3

The broken link is described by dashed line and downstream node of broken link send route maintenance packets towards destination. This describes in figure 4.

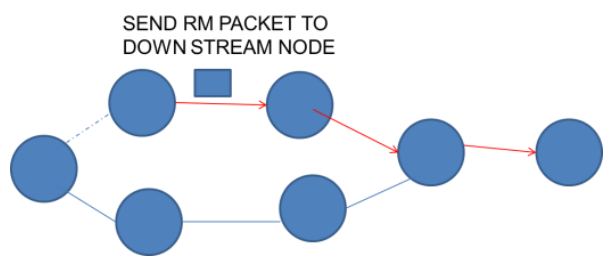

Figure 4

Node receives route maintenance packet and it has another route which can be taken as backup route. Such node is depicted with red colored border in figure 5 .

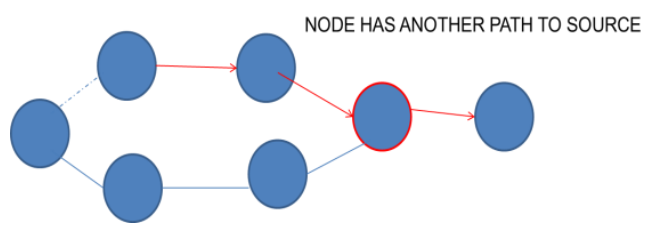

Figure 5

Information about availability of new route from source to destination is conveyed by sending route maintenance packet to source is described in figure 6 .

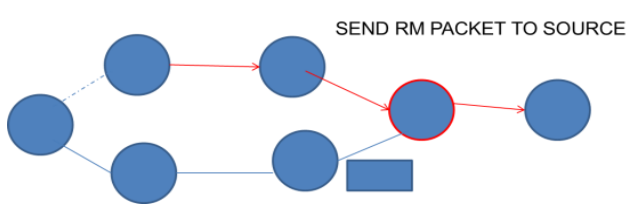

Figure 6

Establishment of new path from source to destination is described by green line in figure 7 .

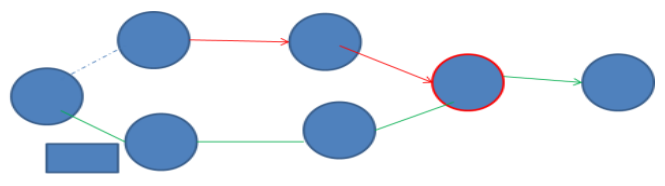

SOURCE IS INFORMED ABOUT ANOTHER ROUTE AND NEW PATH IS ESTABLISHED

\section{PERFORMANCE OF PROPOSED PROTOCOL}

We implemented the AEODV protocol using QUALNET Simulator and they are compared with SARSA-ENERGY AWARE and AODV.

\subsection{SIMULATION PARAMETERS SETING}

Table 1

\begin{tabular}{|l|l|}
\hline Simulation parameter & Value \\
\hline Network Scale & $1000 \mathrm{~m} \times 1000 \mathrm{~m}$ \\
\hline Simulation Time & $1000 \mathrm{~s}$ \\
\hline Number of nodes & 100 \\
\hline Mobility Model & Random Way Point \\
\hline $\begin{array}{l}\text { Maximum Nodes } \\
\text { Velocity }\end{array}$ & $10 \mathrm{~m} / \mathrm{s}$ \\
\hline Pause Time & 0 \\
\hline Traffic Type & $\mathrm{CBR}$ \\
\hline Connections Number & $5,10,15,20$ \\
\hline $\begin{array}{l}\text { Packets Transmission } \\
\text { Rate }\end{array}$ & 4 Packets/s \\
\hline Packet Size & $512 \mathrm{bytes}$ \\
\hline Initial energy & $12 \mathrm{mAh}$ \\
\hline T Sampling Interval & $6 \mathrm{~s}$ \\
\hline Learning Rate $\alpha$ & 0.9 \\
\hline Discount Factor $\gamma$ & 1 \\
\hline Exploration Rate $\varepsilon$ & 0.5 \\
\hline
\end{tabular}

We measured the following performance metrics:

1) Total Packet Received.

2) Average end-to-end Delay (Delay).

3) Throughput.

4) Residual Energy.

\subsection{Total Packet Received}

This describes no of packets received at server side.

Table 2

\begin{tabular}{|c|c|c|c|c|}
\hline & \multicolumn{4}{|c|}{ No of Connections } \\
\hline Protocol & $\mathbf{5}$ & $\mathbf{1 0}$ & $\mathbf{1 5}$ & $\mathbf{2 0}$ \\
\hline AODV & 39649 & 73411 & 107072 & 142135 \\
\hline SARSA & 39641 & 73737 & 107568 & 142326 \\
\hline AEODV & 39644 & 73846 & $\mathbf{1 0 8 5 4 0}$ & $\mathbf{1 4 2 7 3 1}$ \\
\hline
\end{tabular}

Figure 7 


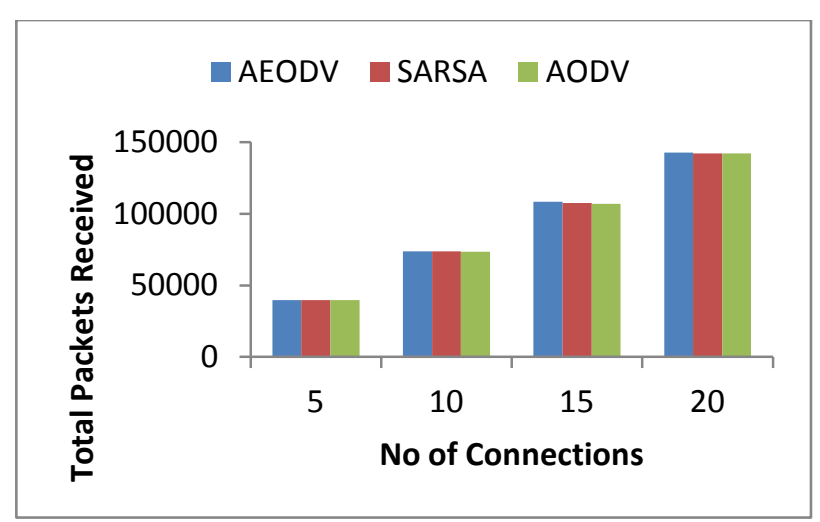

Figure 8

Figure 8 shows increasing no of links increases, AEODV performs better than SARSA and AODV.

\subsection{Average end-to-end Delay (Delay).}

The Average end-to-end delay is defined as the time taken by all packets to reach its destination node after they are generated.

Table 3

\begin{tabular}{|c|c|c|c|c|}
\hline & \multicolumn{4}{|c|}{ No of Connections } \\
\hline Protocol & $\mathbf{5}$ & $\mathbf{1 0}$ & $\mathbf{1 5}$ & $\mathbf{2 0}$ \\
\hline AODV & 0.02311 & 0.04240 & 0.07868 & 0.47678 \\
& & & & \\
\hline SARSA & $\mathbf{0 . 0 2 1 9 3}$ & $\mathbf{0 . 0 3 8 4 9}$ & 0.06443 & 0.36514 \\
\hline AEODV & 0.02298 & 0.04050 & $\mathbf{0 . 0 6 0 6 7}$ & $\mathbf{0 . 1 7 1 3 6}$ \\
\hline
\end{tabular}

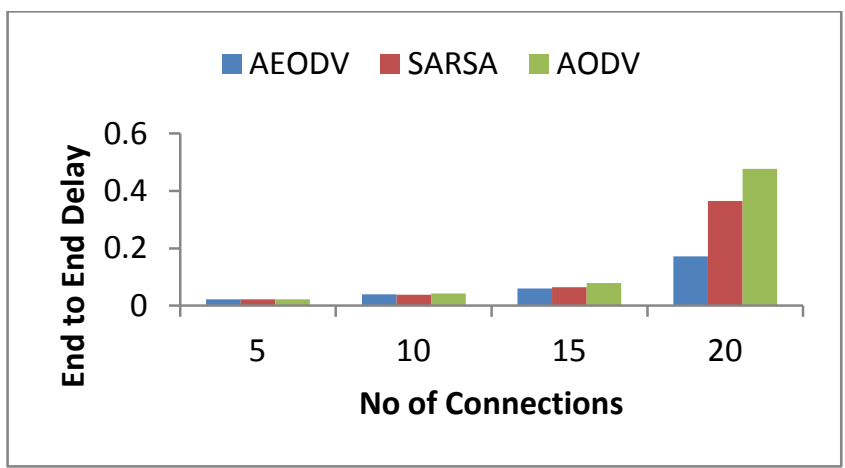

Figure 9

Figure 9 describes that when no of links increases, AEODV has lesser delay than SARSA and AODV.

\subsection{Throughput}

The throughput is the rate of successful message delivery over a communication channel.

Table 4

\begin{tabular}{|c|c|c|c|c|}
\hline & \multicolumn{4}{|c|}{ No of Connections } \\
\hline Protocol & $\mathbf{5}$ & $\mathbf{1 0}$ & $\mathbf{1 5}$ & $\mathbf{2 0}$ \\
\hline AODV & 32538.8 & 30155.4 & 29397.8 & 29083.2 \\
& & & & \\
\hline SARSA & 32524.2 & 30265.6 & 29460.3 & 29271.25 \\
\hline AEODV & 32526 & 30305.2 & 29760.5 & $\mathbf{2 9 4 0 1 . 6 5}$ \\
\hline
\end{tabular}

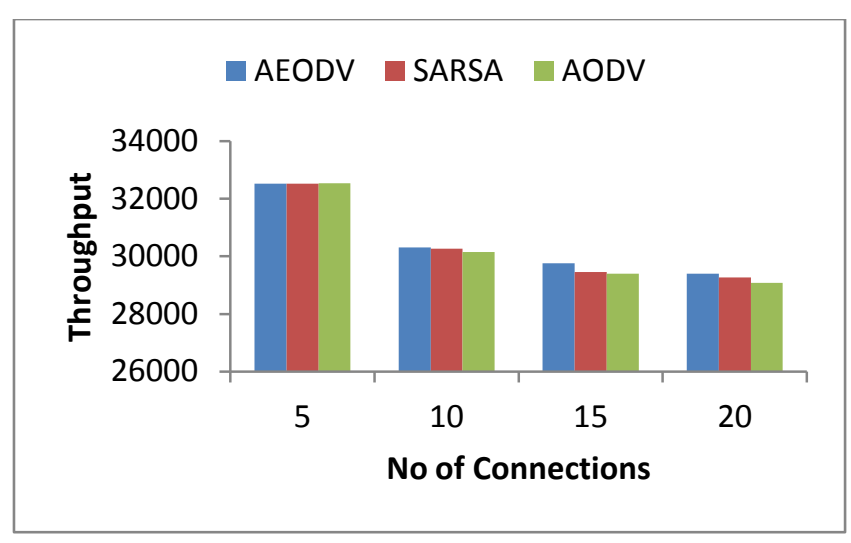

Figure 10

Figure 10 denotes that when no of links increases, AEODV has higher throughput than SARSA and AODV.

\subsection{Residual Energy}

The residual energy is sum of remaining charge for each node's battery.

Table 5

\begin{tabular}{|c|c|c|c|c|}
\hline & \multicolumn{4}{|c|}{ No of Connections } \\
\hline Protocol & $\mathbf{5}$ & $\mathbf{1 0}$ & $\mathbf{1 5}$ & $\mathbf{2 0}$ \\
\hline AODV & 407.9 & 391.45 & 372.19 & 347.36 \\
& & & & 356.11 \\
\hline SARSA & 409.04 & 392.59 & 373.28 & 381.44 \\
\hline AEODV & $\mathbf{4 1 0 . 8 9}$ & $\mathbf{3 9 5 . 4 6}$ & $\mathbf{3 8 4 . 4 7}$ & $\mathbf{3}$ \\
\hline
\end{tabular}

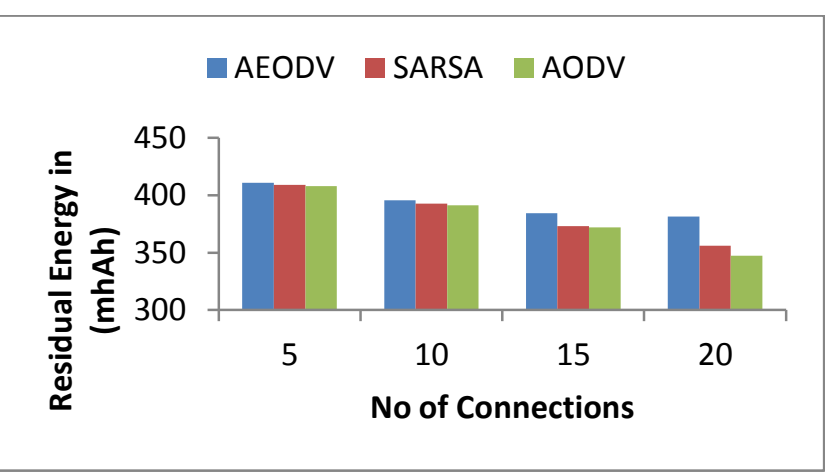

Figure 11

Figure 11 shows AEODV has higher residual than SARSA and AODV at all time.

\section{CONCLUSION \& FUTURE WORK}

All nodes are distributed and there is no centralized mechanism to control them in MANET. SARSA Reinforcement Learning algorithm which provides balanced energy consumption to increase overall network lifetime, but issue of rebroadcasting due to link breakage was still there. It is reason for unnecessary rebroadcasting. Consequence is higher energy consumption. This issue is eliminated using multi-path route maintenance.

AEODV performs well when no of connections are more, but when no of connections are less it performs lesser. So this problem will be solved by adding to or replacing with a new mechanism to MANET's protocols. 


\section{REFERENCES}

[1] Siva C., Murty R., Manoj B.S.: 'Ad hoc wireless networks' (Pearson, 2005)

[2] K. Scott and N. Bambos, "Routing and Channel Assignment for Low Power Transmission in PCS," Proc. Fifth IEEE Int'l Conf. Universal Personal Comm. (ICUPC '96), Oct. 1996.

[3] S. Doshi, S. Bhandare, and T.X. Brown, "An On Demand Minimum Energy Routing Protocol for a Wireless Ad Hoc Network," ACM Mobile Computing and Comm. Rev., vol. 6, no. 3, pp. 50-66, July 2002.

[4] V. Rodoplu and T. Meng, "Minimum Energy Mobile Wireless Networks," IEEE J. Selected Areas in Comm., vol. 17, no. 8, pp. 1333-1344, Aug. 1999.

[5] S. Banerjee and A. Misra, "Minimum Energy Paths for Reliable Communication in Multi-Hop Wireless Networks," Proc. ACM MobiHoc, June 2002.

[6] J. Gomez, A.T. Campbell, M. Naghshineh, and C. Bisdikian, "Conserving Transmission Power in Wireless Ad Hoc Networks," Proc. IEEE Ninth Int'l Conf. Network Protocols, Nov. 2001.

[7] J. Zhu, C. Qiao, and X. Wang, "A Comprehensive Minimum Energy Routing Protocol for Wireless Ad Hoc Networks,” Proc. IEEE INFOCOM, Mar. 2004.
[8] C.K. Toh, H. Cobb, and D. Scott, "Performance Evaluation of Battery-Life-Aware Routing Schemes for Wireless Ad Hoc Networks," Proc. IEEE Int'l Conf. Comm. (ICC '01), June 2001.

[9] A. Misra and S. Banerjee, "MRPC: Maximizing Network Lifetime for Reliable Routing in Wireless Environments," Proc. IEEE Wireless Comm. And Networking Conf. (WCNC '02), Mar. 2002.

[10] Chettibi and S. Chikhi, "An adaptive Energy Aware Routing Protocol for MANETs using SARSA Reinforcement Learning Algorithm", IEEE Conference on Evolving and Adaptive Intelligent Systems (EAIS), pp. 8489, May 2012

[11] R. Sutton and A. Barto, "Reinforcement learning," MIT Press, Cambridge, 1998

[12] C.E. Perkins, E.M. Royer, "Ad-hoc On-Demand Distance Vector Routing", Proceedings of the 2nd IEEE Workshop on Mobile Computing Systems and Applications, Feb. 1999

[13] Mahesh K. Marina and Samir R. Das "Ad hoc on demand multipath distance vector routing" computer Science Department, University of California, Los Angeles, CA 90095-1596, U.S.A., Computer Science Department, State University of New York at Stony Brook, Stony Brook, NY 11794-4400, U.S.A. 